\title{
Towards a quality model for semantic IS standards
}

\author{
Erwin Folmer* \\ University of Twente, \\ Drienerlolaan 5, 7522 NB Enschede, The Netherlands \\ and \\ TNO, \\ Capitool 10, 7521 PL Enschede, The Netherlands \\ E-mail: erwin.folmer@tno.nl \\ *Corresponding author

\section{Joris van Soest} \\ University of Twente, \\ Drienerlolaan 5, 7522 NB Enschede, The Netherlands \\ E-mail: j.1.m.vansoest@student.utwente.nl
}

\begin{abstract}
This research focuses on developing a quality model for semantic information system (IS) standards. A lot of semantic IS standards are available in different industries. Often these standards are developed by a dedicated organisation. While these organisations have the goal of increasing interoperability, there is no way to determine the quality of such a standard. This research will provide quality attributes relevant to semantic IS standards. A theoretical grounded model is created and validated by 19 experts through a survey. Based on these findings, a quality model to assess the quality of semantic IS standards has been constructed.
\end{abstract}

Keywords: quality model; semantic IS standard; interoperability; information; quality.

Reference to this paper should be made as follows: Folmer, E. and van Soest, J. (2012) 'Towards a quality model for semantic IS standards', Int. J. Information Quality, Vol. 3, No. 1, pp.28-48.

Biographical notes: Erwin Folmer received his Masters degree in Technical Business Administration from the University of Twente. In 1999, he started working for KPN Research as an Innovator for designing order entry and billing systems for new KPN services such as ADSL. From 2001, he joined TNO. Since then, he has been involved in the standardisation and interoperability research with a special interest in semantic standards. In 2009, he joined the University of Twente part-time to embark on a PhD research programme on standardisation, while continuing his work for TNO.

Joris van Soest studied Business Information Technology at the University of Twente, The Netherlands. His special interests are related to semantic technologies and semantic standards. 


\section{Introduction}

With the introduction of XML and the internet, e-business became available for many companies. Much focus is nowadays on the concept of inter-organisational interoperability: the ability of two or more social-technical systems to exchange information, to interpret the information that has been exchanged and to act upon it in a appropriate and agreed-upon matter (Rukanova, 2005).

Research has shown that a lack of interoperability costs the automotive industry in the USA an estimate of \$1 billion per year and a delay of two months in the introduction of new models (Brunnermeier and Martin, 2002). Standardisation is a way to achieve interoperability. A standard, in the simplest sense, is an agreed-upon way of doing something (Spivak and Brenner, 2001). Semantic information system (IS) standards are used to communicate and cooperate with partners, suppliers or customers in an efficient and effective way. These semantic IS standards describe the meaning of information and syntax of messages that are exchanged. A semantic IS standard is a mean to achieve the goal of interoperability. The extent to which a semantic IS standards is capable of providing an effective contribution to this interoperability can be described as the fitness for use. So a qualitative good standard is able to achieve a high level of interoperability.

Although these standards are usually developed with the best intentions, they often have quality issues like difficult to understand, multiple interpretations, etc. (Folmer et al., 2010a). Hardly any study has been done to determine which quality aspects increase interoperability. This study will focus on developing a quality model for semantic IS standards.

Quality of semantic IS standards is strongly related to information quality. The main distinction is that the academic area of information quality often is focused on the quality of information within an organisation, while on the other hand the quality of information exchanged between organisations is often related to the area of semantic IS standards. In other words quality of semantic IS standards deal with inter-organisational information quality. Semantic IS standards are the traditional mean for data integration within interorganisational value chains. These inter-organisational value chains might be related to e-business, or more specific related to e-health, e-learning, etc.

\subsection{Background}

Most of the IT-standards are developed outside traditional standards developing organisations (like ISO or CEN), in so called industry specific consortia (like W3C or OASIS). Semantic IS standards are even a step further, they are often developed in a separate organisation dedicated to one specific industry standard. An example is the HRXML standard developed by the HR-XML Consortium.

Because there are so many different consortia, the quality of a standard can differ quite a lot between them. It is remarkable that little is known about quality of semantic IS standards. If standards are being developed to increase interoperability, the degree in which interoperability can be achieved will most likely be influenced by the quality of the standard. A research among 34 standard developing organisations (SDOs), including international standards like XBRL, HR-XML, ACORD, HL7 and national standards like SETU, StUF, Aquo, shows that more than $90 \%$ of these organisations think that the quality can be improved (Folmer et al., 2010b). A large majority thinks an improvement 
of their standard will contribute to better interoperability. It is, however, difficult to improve quality if the quality is not known. More than $80 \%$ of the questioned SDOs would like to use a tool to assess the quality of their standard if it is available.

\subsection{Problem statement}

To date there does not exist a quality model to assess the quality of semantic IS standards. While most standards are developed to increase the interoperability in specific domains, there is a lack of methods to assess the quality of these standards. In a business environment where there is an increasing exchange of information, it becomes more and more important to develop standards of high quality to improve efficiency and effectiveness of inter-organisational data integration.

\subsection{Research questions}

Since there is a need for a quality model for semantic IS standards, more research on this specific area is required. The overall goal is to build a quality model for semantic IS standards. To achieve this goal, the following research questions are relevant:

- What structured set of quality attributes determine the quality of a semantic IS standard?

- What can we learn from other disciplines, like software engineering or product engineering?

The results will be a structured list of quality attributes that are applicable to the domain of semantic IS standards. A description will be made for each quality attribute including a definition. A validation will be performed to determine the extent of usefulness to practice of this model.

The outline of this research is as follows. In Section 2, the research methodology will be explained. To create a model to assess the quality of semantic IS standards, we first take a look at the literature. A literature study has been conducted to find quality attributes which can determine the quality of a semantic IS standard (Section 3). A model is constructed based on the findings in literature (Section 4). This model is validated through a survey (Section 5). Based on the finding of the survey, a second improved quality model has been constructed (Section 6). The paper ends with several conclusions and future outlook (Section 7).

\section{Research approach}

This research is conducted using the design science principle as explained by Hevner et al. (2004). "Design science addresses research through the building and evaluation of artifacts [...]" (Hevner et al., 2004). This process is inherently iterative, and consists of build and evaluation steps. This cycle is repeated until the appropriate business needs are satisfied. The artefact is the quality model for semantic IS standards. Within the build phase we will use theories available in literature to create a first model, the artefact. We will evaluate this model (the artefact) through a survey. These two steps complete the first iteration of the design cycle. The design artefact becomes more relevant and valuable 
with each iteration (Hevner et al., 2004). After our first iteration, a second iteration is started, consisting only of build phase. The evaluation results are used to refine the artefact, our end result.

\section{Literature review}

Recent study has identified a research gap on quality of transactional standards (Folmer et al., 2009). A systematic literature study was conducted that covered the top 25 journals of ISs. Since there is no quality model specific for semantic IS standards, we need to look at literature that might have some parts in common. Three main research areas have been looked into; product quality, data- and information-quality and ISs/software quality. These areas all have a history in quality research and have commonalities with semantic IS standards.

Most notable authors in the field of product quality we looked in to are: Crosby (1979) and Garvin (1984).

There is lots of research about quality conducted in the field of ISs/software quality. Arguably most famous is the work of Cavano and McCall (1978), McCall et al. (1977), Boehm (1973), ISO 9126, DeLone and McLean (2003). But many others have deliverable valuable work as well like Delen and Rijsenbrij (1992), Grady (1992; FURPS model), Dromey (1995, 1996), Dedeke (2000) and O'Brien et al. (2007). Authors in the field of data quality and information quality are, amongst others, Wand and Wang (1996), Wang and Strong (1996), Katerattanakul and Siau (1999), Alexander and Tate (1999), Shanks and Corbitt (1999), Naumann and Rolker (2000), Zhu and Gauch (2000) Kahn et al. (2002), and Stvilia et al. (2007).

While their application domains might differ, all used definitions or classification of quality are more often similar, then different. We summarised the quality attributes from this vast amount of literature. Table 1 shows this summary including the originating discipline (the 3 columns).

\section{Draft quality model}

Based on all the quality aspects we found in the previous section we continued the first build phase. A first selection of attributes that are relevant in assessing quality of semantic IS standards was done within the integrate project, by having expert sessions select and discuss the most relevant attributes. The outcome, the draft model, is heavily inspired on the ISO 9126 model, especially the categorisation. The ISO 9126 model is a popular framework and is commonly used to assess the quality of software. The attributes present in the ISO 9126 model are well defined and provided us with the base of our quality model for semantic IS standards. All attributes have been selected that were labelled relevant in relation to quality of semantic IS standards within the Integrate project. However, within this project the attribute 'acceptance' was added, although no traces of this attributes in the literature was found. Both the use of ISO 9126 as well as the selection of the quality aspects is somewhat arbitrary, but this limitation has been overcome by the survey as evaluation. 
Table 1 List of quality attributes including sources

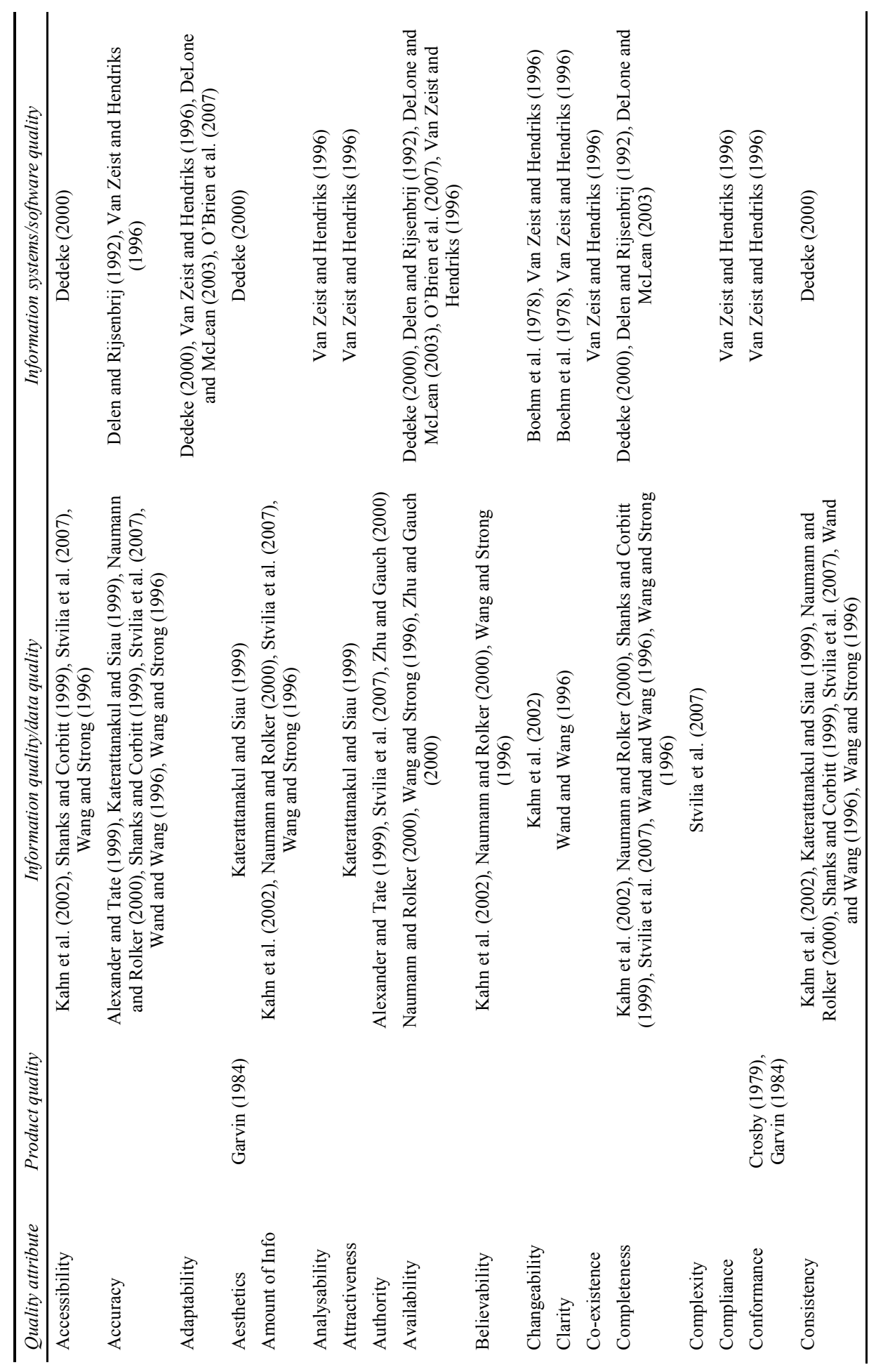


Towards a quality model for semantic IS standards

Table 1 List of quality attributes including sources (continued)

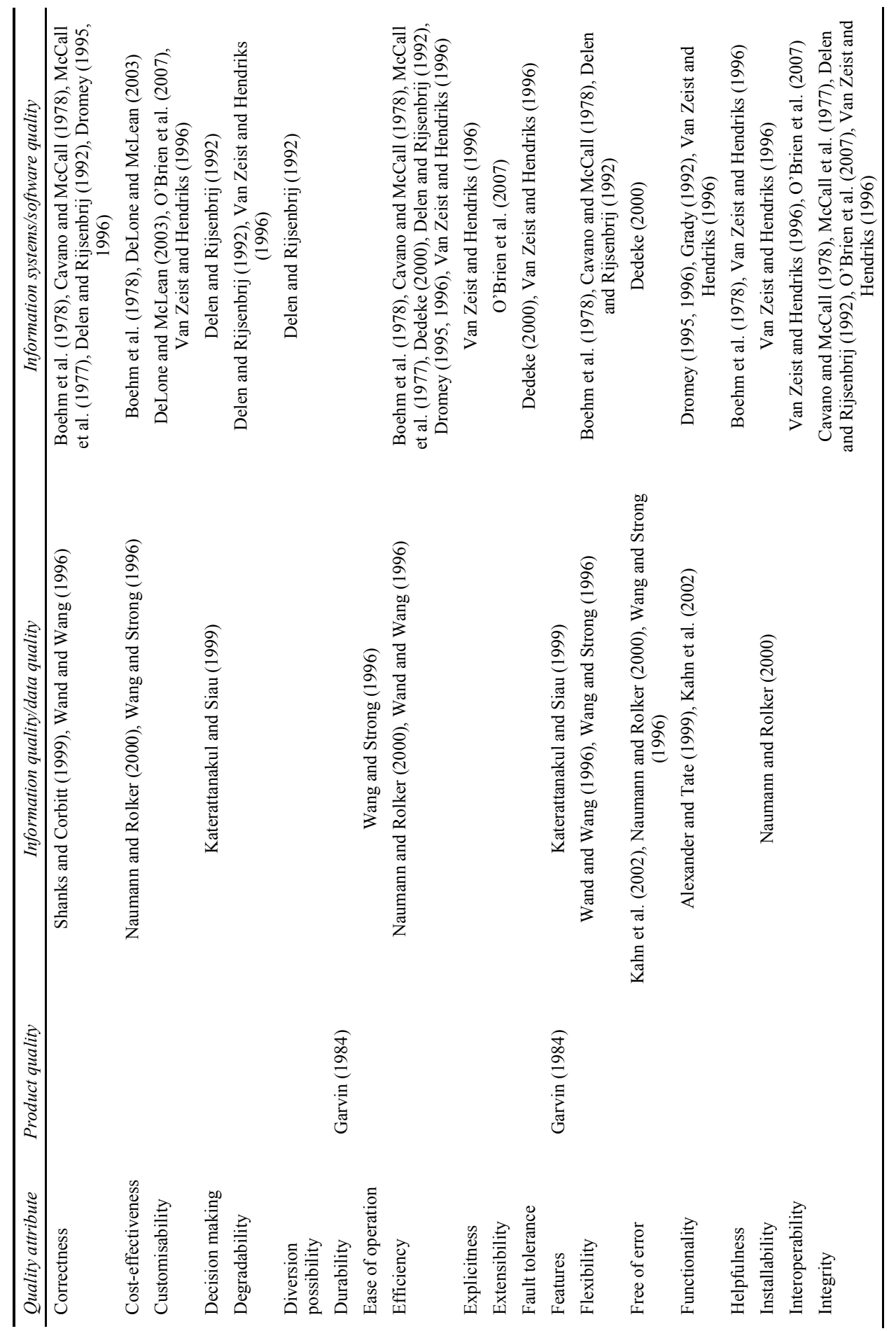


Table 1 List of quality attributes including sources (continued)

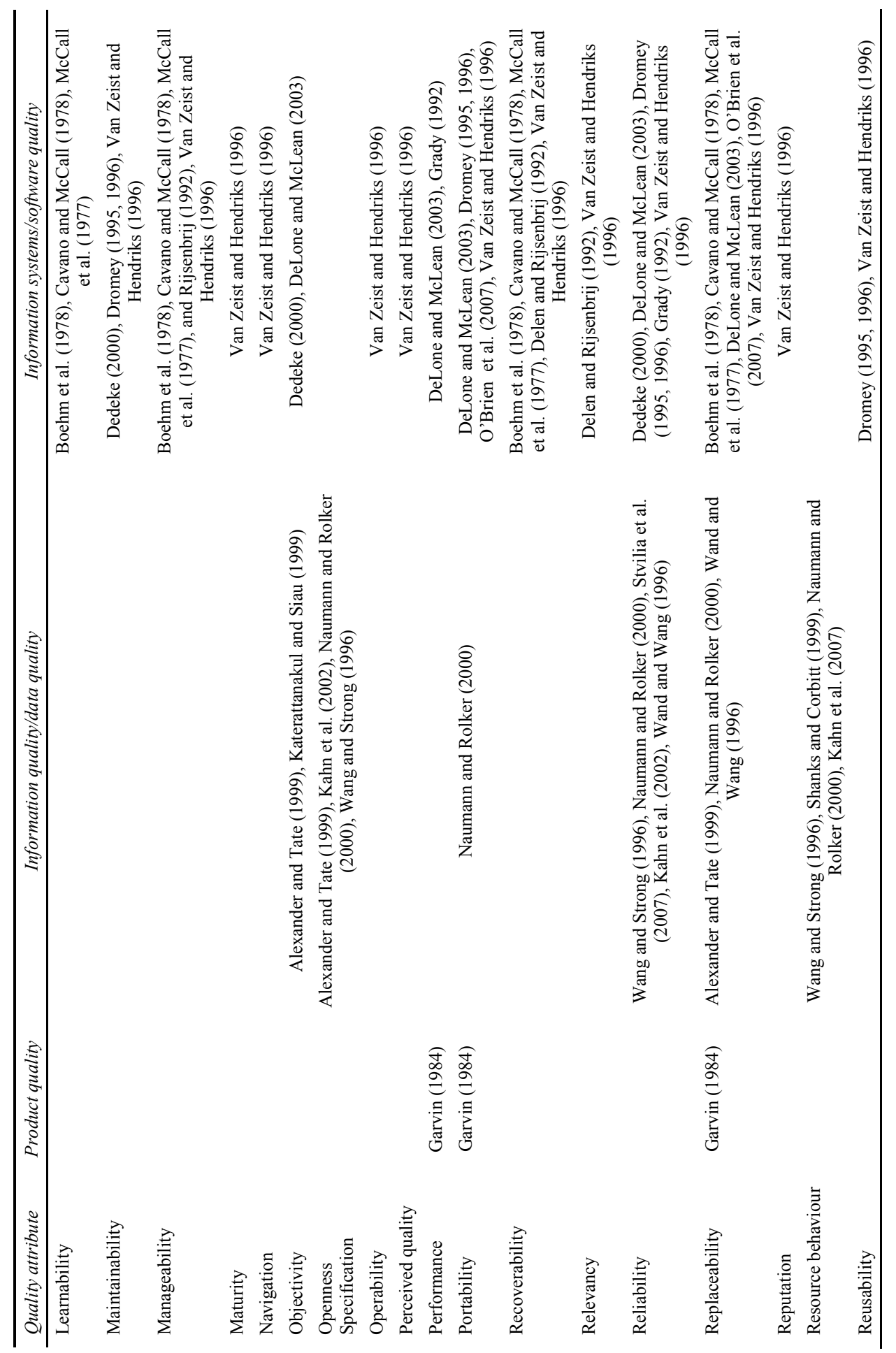


Table 1 List of quality attributes including sources (continued)

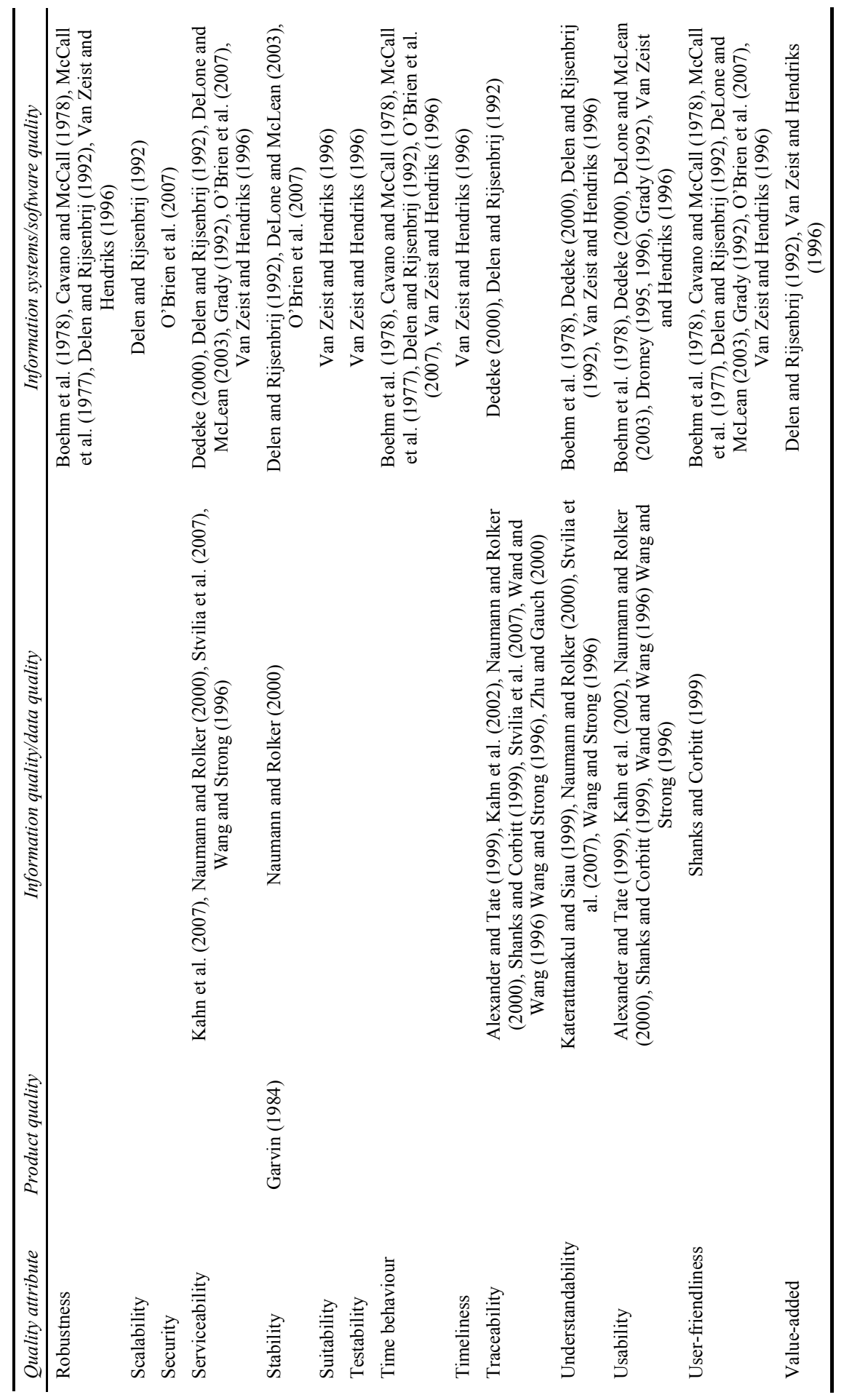




\subsection{Categorisation}

The categories, in line with ISO 9126, included in the draft model are: functionality, reliability, usability, portability and maintainability. The categorisation makes it possible for the user to select parts of the quality model based on its specific needs. Adoptability and openness are two categories that were added as category. The openness category was added because this is nowadays seen as important aspect of a standard although it is related to the standard development organisation. However, it is also seen as indicator for the quality of the specification. The model is depicted in Figure 1.

Figure 1 Draft quality model

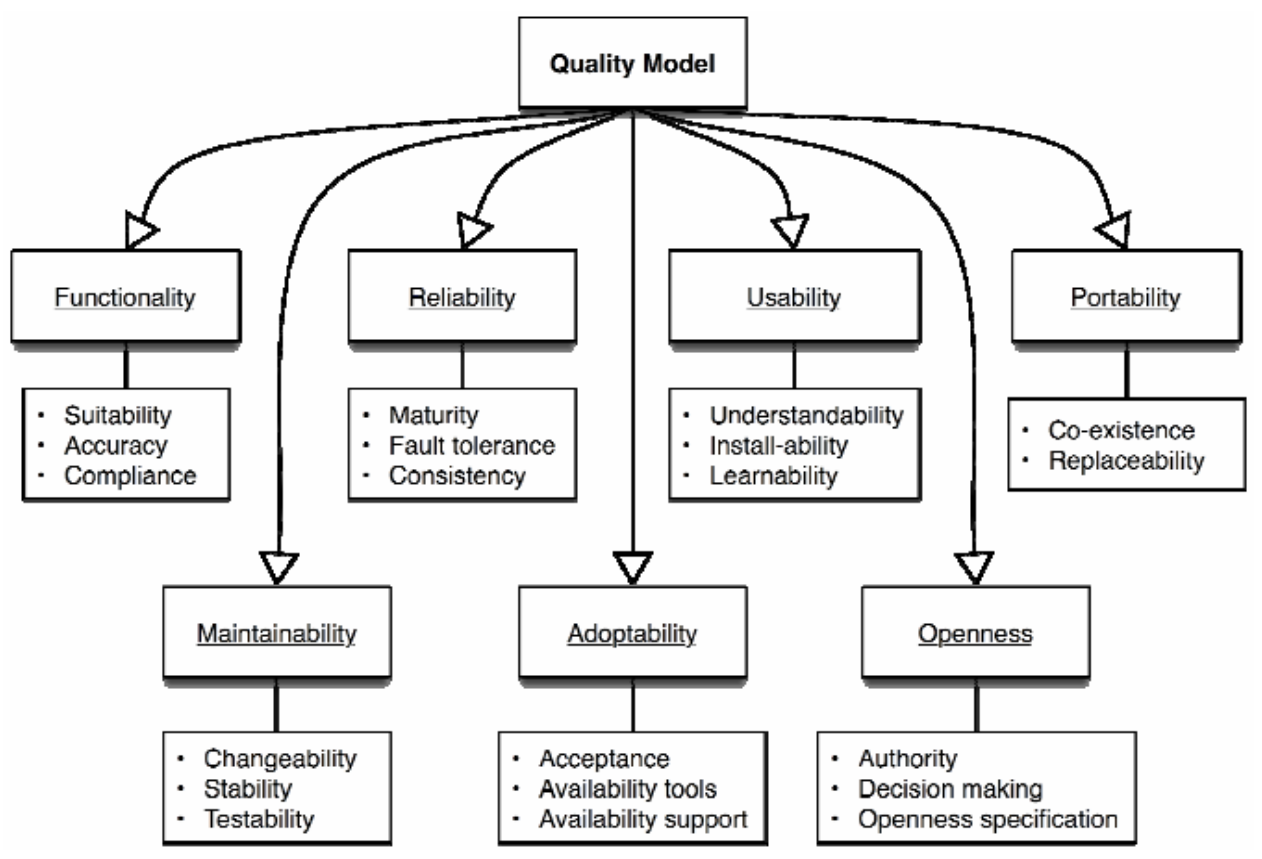

\subsection{Elements of draft model}

The definitions of each attribute will be followed by an application of that attribute to the field of semantic IS standards.

- Suitability [definition adapted from Van Zeist and Hendriks (1996)]:

The capability of the standard to provide an appropriate set of functions for specified tasks and goals. Standards are being used to overcome interoperability issues.

- Accuracy [definition adapted from Van Zeist and Hendriks (1996)]:

The capability of the standard to provide the right or agreed results or effects with the needed degree of precision. Does the implementation of the standard do what it is supposed to do? Does it live up to the expectations? 
- Compliance [definition adapted from Van Zeist and Hendriks (1996)]:

The capability of the standard to adhere to standards, conventions or regulations in laws and similar prescriptions. This can come from government or the industry. Financial reports are a good example. To what extent are these aspects covered within the standard?

- Maturity [definition adapted from Van Zeist and Hendriks (1996)]:

The capability of the standard to avoid failure as a result of faults in the standard. When there are not many bugs in the standard, errors will not likely occur. The amount of unsolved bugs or the amount of changes in a release might be a good indicator for this. If the standard is mature, often there is a stable release schema for new versions.

- $\quad$ Fault tolerance [definition adapted from Van Zeist and Hendriks (1996)]:

The capability of the standard to maintain a specified level of performance in cases of faults occurring in the implementation. The amount of manual work needed for correcting an error can be a good indicator. Can the implementation continue to work with the error?

- Consistency [definition adapted from Stvilia et al. (2007)]:

The extent to which similar attributes or elements of an information object are consistently represented using the same structure, format, and precision. Inconsistency will most likely lead to errors in use or implementation.

- Understandability [definition adapted from Van Zeist and Hendriks (1996)]:

The capability of the standard to enable the user to understand whether the standard is suitable, and how it can be used for particular tasks and conditions of use. Is all the information easy to read? Complex documents will not lead to better implementations. Readability scores can be a good indicator.

- Install-ability [definition adapted from Van Zeist and Hendriks (1996)]:

The extent to which the standard can be implemented easily. Is the standard easily installed into existing ISs or organisations?

- Learnability [definition adapted from Van Zeist and Hendriks (1996)]:

The capability of the standard to enable the user to learn its application. The time needed for a user to learn the use or implementation of the standard.

- Co-existence [definition adapted from Van Zeist and Hendriks (1996)]:

The capability of the standard to exist next to other standards. Will a standard function properly next to another standard, set up for the same goal? Is it possible to access the same information, or does the information use different naming for example?

- Replaceability [definition adapted from Van Zeist and Hendriks (1996)]:

The capability of the standard to be used in place of another specified standard for the same purpose in the same environment. Is it possible to replace the current 
standard with a newer version without much hassle? Does the standard provide backwards compatibility?

- Changeability [definition adapted from Van Zeist and Hendriks (1996)]:

The capability of the standard to enable a specified modification to be implemented. Does the standard provide possibilities for committing changes to the standard? Does the standard provide the option to add localisation functions or code-lists? How long does it take to change something in the standard?

- Stability [definition adapted from Van Zeist and Hendriks (1996)]:

The capability of the standard to avoid unexpected effects from modifications of the standard or environment. New versions emerge over time, as well as new hardware or infrastructure. Does the standard keep its level of function after changes?

- Testability [definition adapted from Van Zeist and Hendriks (1996)]:

The capability of the standard to enable implementations to be validated. Is there a way to test an implementation? The availability of reference implementation might help. Is there certification?

- Acceptance:

The extent to which the standard is used and supported by different kind of stakeholders. How well is the standard used in the target domain? A measurement can be the market share of a standard.

- Availability tools:

The extent to which the standard provides tools for implementation. Implementing the standard should be as easy as possible. Additional tools to support the implementation should increase it use. Does the SDO provide methods to let the standard communicate with other software products?

- Availability support:

The extent to which knowledge and support is available. To use a certain standard knowledge is needed to implement it successfully. Is there enough knowledge and support available? How fast do you get response from the support department? Is there some external consultancy available for this standard?

- Authority [definition adapted from Stvilia et al. (2007)]:

The degree of reputation of the standard in a given community or business area. Some standards are highly valued by certain users. This might be because the standard is of better quality, or because of the reputation of the standard development organisation.

- Decision making [definition adapted from Delen and Rijsenbrij (1992)]:

The organisational characteristics of the SDO and the way decisions are being made. Is there consensus decision making, or majority voting, or anything else? 
- Openness specification:

The extent to which the standard provides free to use specifications. Is the specification available for everyone without additional costs or efforts?

\section{Survey}

Evaluation is a crucial component of the research process (Hevner et al., 2004). It provides valuable feedback to the development of the artefact. "A design artifact is complete and effective when it satisfies the requirements and constraints of the problem it was meant to solve" (Hevner et al., 2004). The evaluation is being conducted as final step in the first design cycle iteration. Evaluations can take place in different ways, some examples of evaluations are; surveys, experiments, simulations or case studies. In this research, a survey was selected. A survey has some major advantages for our purpose of getting feedback on our quality model. A survey is conducted in the field, not in a laboratory. This will ensure the information gathered will be relevant for the next iteration in the design cycle as it contains information about the business context it is meant to be used in. Another advantage of a survey is that you do not have control over the participant. There is little interference which will provide us with honest, unbiased answer to the questions.

This survey had two main goals; first to check what the experts think are important quality attributes for semantic IS standards, second to check if our chosen quality attributes are relevant for assessing the quality of semantic IS standards. These two parts were clearly separated in the survey to ensure our model did not bias the respondents. The model was only introduced after the first part of the survey was finished. The first part of the survey consists of our large list of quality attributes. The following question was asked:

Q1 Which elements do you think are in some way relevant for assessing the quality of a semantic IS standard?

The choices were our previously found 70 quality attributes, presented with a checklist where multiple answers were possible. A pop-up window was provided with a list of all quality attributes and definitions.

The second part of the survey is intended to validate the model, by determining if the selected quality attributes should be included in the model. Furthermore, a question was asked about how to categorise the quality attributes that were selected in the first part of the survey but were not included in the model.

First, a definition of the category was given, and then the selection of quality attributes was listed. After giving a definition of the selected quality attribute we asked the following question per attribute, on a five-point Likert scale:

Q2 Do you agree that SUITABILITY should be included in the model?

We repeated the same type of question for every quality attribute in each category, including a definition of that specific attribute. The final question for each category was:

Q5 Which of the your previously selected attributes should be added to this category? 
Here, the possible answers were the selected attributes from the first part of our survey (Q1), presented in a multi-selection checkbox, again with the possibility to have a look at the definitions. These questions were repeated for each of the seven categories. Finally, several questions were asked about the respondents, including their experience. The complete set of information about the survey setup and the results is due to space limitations not listed within this paper but is available by contacting the authors.

\subsection{Results}

We selected 27 experts mainly from TNO, University of Twente and Novay, who have participated in research about semantic IS standards. These have been invited to participate in this survey. A total of 19 complete responses were gathered, which resulted in a response rate of $70 \%$. Most of the respondents work at research institutions or universities, while others have been involved in the creating process of a semantic IS standards. The years of experience in the field of semantic IS standards varied between 1 year and 25 years. Of the respondents, $73.7 \%$ had more than three years experience. Among them, $31.6 \%$ considered themselves to be an 'expert', $57.9 \%$ 'average' and $10.5 \%$ a 'beginner'.

Table 2 Survey results, Part 1

\begin{tabular}{lcccc}
\hline Quality attribute & Count & Percent $\%$ & Present in draft model \\
\hline Consistency & 16 & $84.2 \%$ & Yes & No \\
Interoperability & 14 & $73.7 \%$ & & \\
Openness specification & 13 & $68.4 \%$ & Yes & No \\
Adaptability & 12 & $63.2 \%$ & & No \\
Correctness & 12 & $63.2 \%$ & & No \\
Reusability & 11 & $57.9 \%$ & & No \\
Completeness & 11 & $57.9 \%$ & & No \\
Accessibility & 11 & $57.9 \%$ & & \\
Maintainability & 11 & $57.9 \%$ & Yes & \\
Availability & 11 & $57.9 \%$ & Yes & \\
Accuracy & 11 & $57.9 \%$ & Yes & No \\
Understandability & 10 & $52.6 \%$ & Yes & No \\
Usability & 10 & $52.6 \%$ & Yes & \\
Efficiency & 10 & $52.6 \%$ & & \\
Free of error & 10 & $52.6 \%$ & & \\
Testability & 10 & $52.6 \%$ & Yes & \\
$\ldots$ & $\ldots$ & $\ldots$ & & \\
\hline
\end{tabular}

- $\quad$ Part 1

At the first part of the survey, consistency, interoperability and openness specification were considered the most relevant for assessing the quality of semantic IS standards with a score of respectively 16, 14 and 13 at the first question. See Table 2 for a summary of the results. All attributes were selected at least one time, 
except 'Attractiveness', which was not selected a single time. The top 16 answers contained 8 quality attributes which are also present in our draft model.

- $\quad$ Part 2

Table 3 shows the (most significant) results gathered from the second part of the survey. A Cronbach's alpha was calculated on the questions regarding the specific quality attributes used in the model. Cronbach's alpha is a measure of how well individual variables vary, indicating the reliability of the single factor representing the multiple individual variables. Since the individual variables all measure the same construct, namely the quality of semantic IS standards, it is possible to calculate this alpha. The Cronbach's alpha is 0.846 which is considered as very good.

Table 3 Survey results, Part 2

\begin{tabular}{llcc}
\hline Question & Mean & Std. deviation \\
\hline Q2 & Suitability & 4.21 & 1.032 \\
Q3 & Accuracy & 3.63 & 1.212 \\
Q4 & Compliance & 3.95 & 1.177 \\
Q6 & Maturity & 3.53 & 1.172 \\
Q7 & Fault tolerance & 3.00 & 1.085 \\
Q8 & Consistency & 4.68 & 0.582 \\
Q10 & Understandability & 4.16 & 1.259 \\
Q11 & Install-ability & 3.68 & 1.108 \\
Q12 & Learnability & 2.89 & 1.197 \\
Q14 & Co-existence & 3.47 & 1.124 \\
Q15 & Replaceability & 3.61 & 0.979 \\
Q17 & Changeability & 4.00 & 0.745 \\
Q18 & Stability & 3.63 & 0.895 \\
Q19 & Testability & 3.68 & 1.376 \\
Q21 & Acceptance & 3.89 & 0.875 \\
Q22 & Availability tools & 3.58 & 1.071 \\
Q23 & Availability support & 3.89 & 0.937 \\
Q25 & Authority & 2.74 & 0.991 \\
Q26 & Decision making & 4.05 & 1.177 \\
Q27 & Openness specification & 4.21 & 1.273 \\
\hline
\end{tabular}

At the functionality category, six respondents chose completeness and accuracy as to be added to this category. Other attributes, selected more than three times, were the attributes already present in the model.

Free of error and correctness scored respectively 7 and 4 times within the reliability category. Remarkably 'fault tolerance' has one of the lowest means (3.00) and only 1 respondent thinks it should be added to the reliability category. Fault tolerance is also once selected in relation to the functionality category, and it is absent at all other categories. 
At the usability category learnability has a mean of 2.89 which is also one of the lowest, only authority (within openness category) scores even lower. Install-ability scored a mean of 3.68 , but was only selected one time in the question about adding it to this category. Accessibility, not present in the model, was selected five times to be added to this category, the same amount as understandability and usability which were already present in the model.

Co-existence and replaceability both scored quite well at their individual questions, respectively 3.47 and 3.61. Remarkable to note was that the respondents that selected those two attributes in the first part of the survey, a minimum number of respondents chose co-existence (2) and replaceability (1) to be added to the category of portability. adaptability (4) and interoperability (3) have been more often selected.

Within maintainability, changeability scored one of the highest with a mean of 4.00 and a low standard deviation (0.745), but was only selected 5 times at the first part of the survey, and 2 times to be added to this category.

Openness specification was chosen five times to be added to the adoptability category and four times to be added to the openness category, with $50 \%$ and $60 \%$ of the respondents selecting that attribute.

\subsection{Discussion}

Half of the attributes present in our model have also been selected by the participants. The attributes which were selected by more than half of the participants are candidates to include in the second quality model. The results of the second part of the survey learned us that four attributes (fault tolerance, learnability, authority and co-existence) scored a mean lower then 3.5 (see Table 3). All other attributes were higher with a peak of 4.65 of consistency. This is an indicator that our selected attributes (minus those four) present in the model are contributing to the goal of assessing the quality of semantic IS standards, at least according to the experts.

Remarkably not all attributes selected in the first part of the survey, and present in our model, returned at the specific categories. An explanation of this can be that some respondents did not find it necessary to add the already present attribute to the category. It can be seen logical that when a respondent agreed to include an attribute to the model just a few questions before, he did not want that attribute to add somewhere else, so that might have been a reason not to select that answer. Other remarks that were given by the respondents shows the need of avoiding complexity:

"Please keep your model small and simple" and "Don't make the model to complex."

Furthermore, a remark was given about our categorisation:

"[..] All attributes are correct and need to be considered, but from different perspectives. My advice is to structure them according to these perspectives [specification, organizational aspects, adoption, implementation aspects]."

A possible explanation to why the respondents did not select a certain attribute at the first part of the survey, and valued the attribute quite high at the individual question within the second part, might be that the respondent did not read the definition. At the first part of the survey the definition list provided by a button. A list of 70 attributes with definitions emerged in a pop-up window. Although no evidence was found, it could be that some 
participants did not press the button to view the definitions. It is possible the participants saw the definition at the corresponding page for the first time. This might explain the lack of choices in the first part, and high score at the individual questions. For example, 'decision support' might have an unknown definition to the respondents at the beginning of the survey and based on that it was not selected, but when they were forced to read the definition in the second part, it was considered a good attribute. This line of thought is supported by several remarks from the respondents:

\footnotetext{
"The first list contains much too much overlap in definitions"

"[...] So many [attributes] and some seem to be overlapping."

"Due to the large list of possible quality aspects its sometimes different to remember their definitions."
}

\section{Final quality model}

Based on the results and feedback of the survey, we started a second iteration of the design cycle. This resulted in an adjusted list of definitions, specific for semantic IS standards. Some attributes were combined to reduce the ambiguity and overlap. After the new definitions-list was created, a second model was built. Feedback from the survey was included in the new model. This resulted in a reduction from the initial 70 attributes used in our first model, to 35 newly defined attributes.

In the process of grouping and re-defining the attributes, we categorised the new attributes into three new and different categories inspired by the respondents; Specification, Organisational aspects, Implementation. It provides a separation of concerns which can be useful in practice. If someone wants to compare quality attributes associated to the implementation of the standard in different products, you only have to look at the implementation category.

- The specification category is everything that is about the specification. A good rule of thumb is looking at it as a manual for the standard. The specification handles all the elements which can be seen as 'the product'.

- The organisational aspects category is about the control of the standard. It defines how the standard is originated and how the process of development and maintenance is arranged.

- The final category is the implementation category. Here are all the attributes related to the implementation of the specification. It is related to practice, when a specification is used and a standard is functioning in a certain (business) environment.

After the new categorisation and definitions were made, the next step was to update our initial model. The quality attributes from our draft model were included in the second model, except fault tolerance, learnability, authority and co-existence. These four attributes were left out based on the results of the survey. Results of the first part of the survey provided us with the addition of the following attributes to the model: interoperability, correctness, completeness, adaptability, reusability, accessibility, availability, free of error, extensibility. 
44 E. Folmer and J. van Soest

Table 4 Quality attributes for semantic IS standards

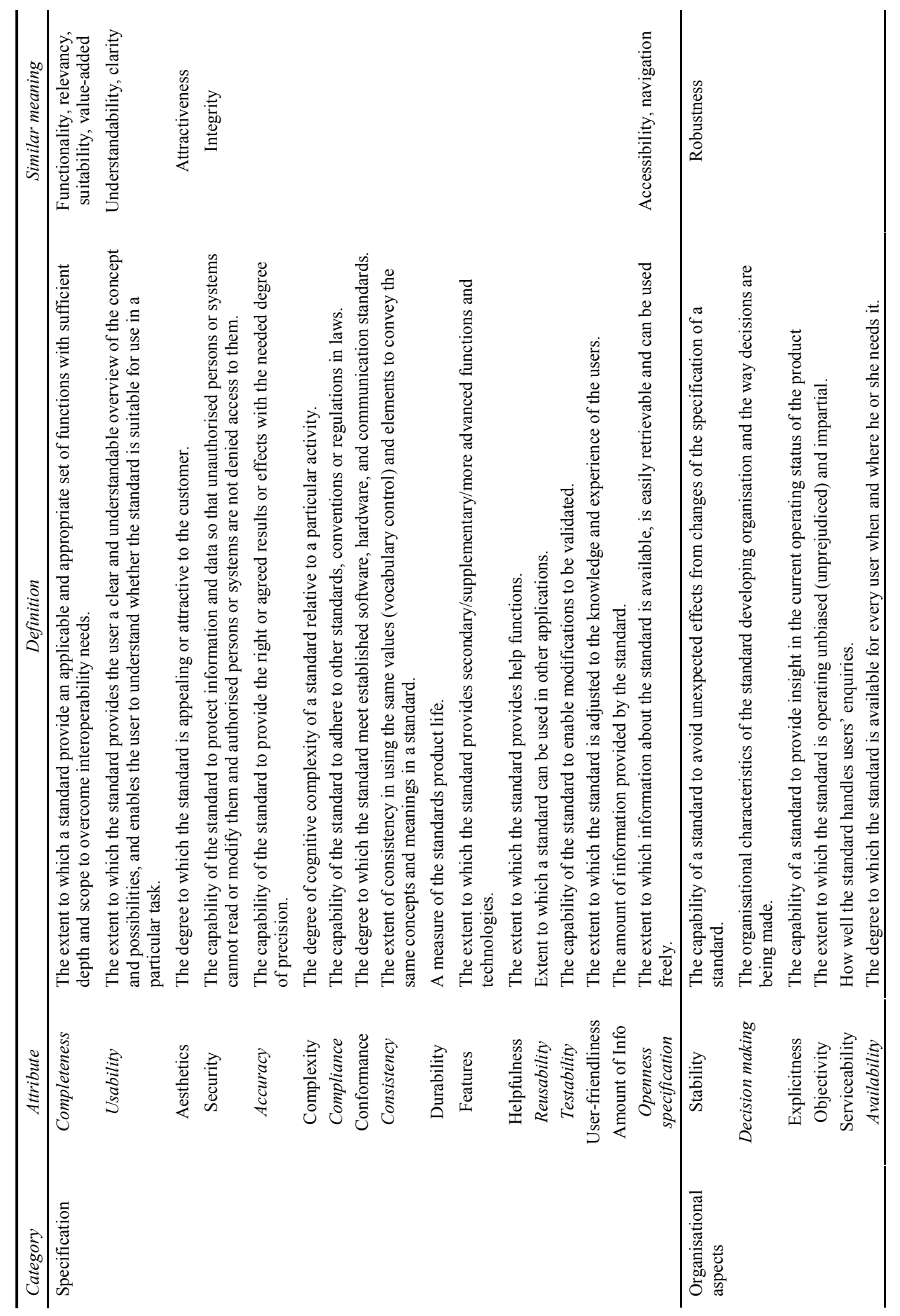


Towards a quality model for semantic IS standards

Table 4 Quality attributes for semantic IS standards (continued)

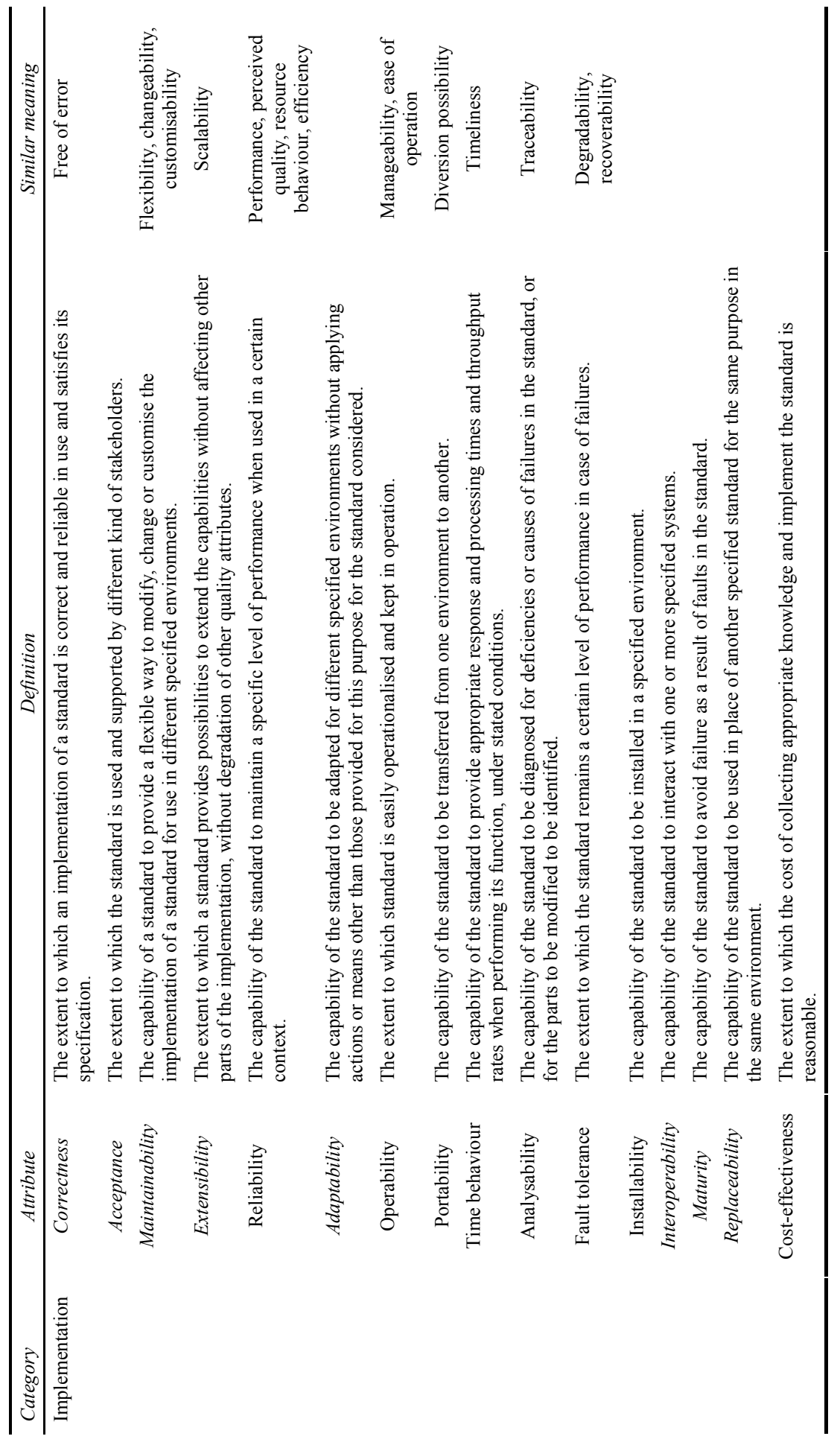


Figure 2 Quality model

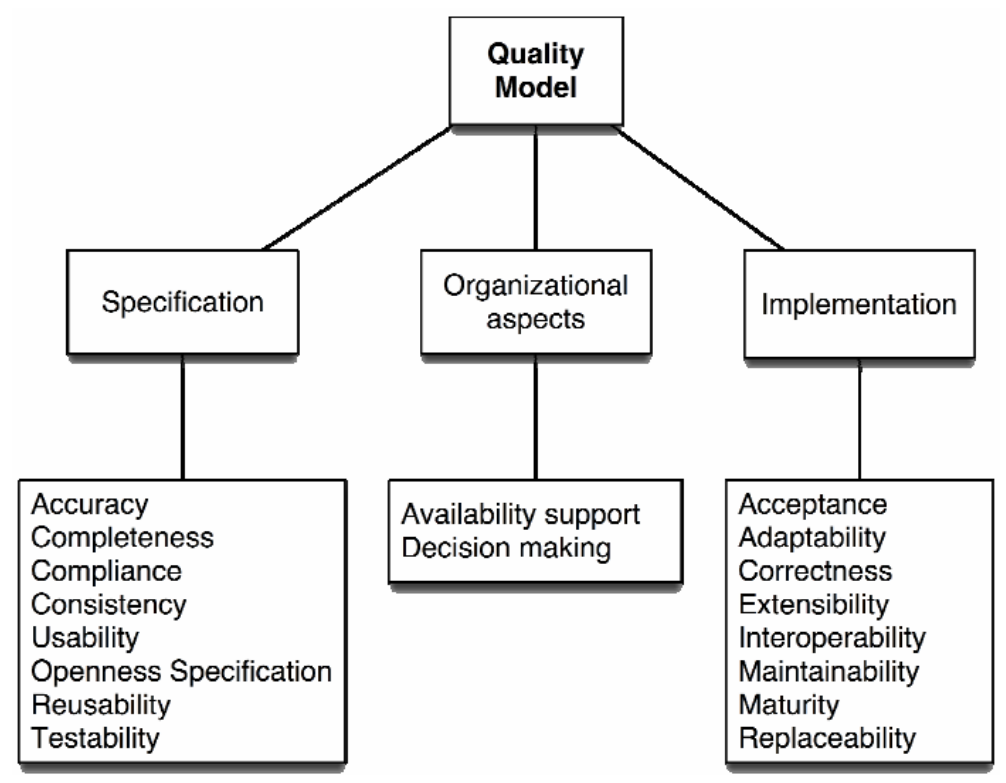

These attributes were selected by more than half $(52.9 \%)$ of the respondents. The next step was to look at the newly created definitions list and merge some attributes to lower the requested complexity of the model. 'Correctness' and 'free of error' is an example of two attributes which was merged into one. The resulting quality model is presented in Figure 2.

The grouping and re-defining of the attributes was done in small iterations. Attributes with similar meaning were grouped together. Each group (or single attribute) was assigned to a category: specification, organisational aspects, or implementation category. This process was repeated until every attribute was assigned to the new categories. For example, the attributes maintainability, flexibility, changeability and customisability were combined into one attribute 'maintainability'. The definition was adapted accordingly and shows traces to the broadened meaning of maintainability: 'The capability of a standard to provide a flexible way to modify, change or customise the implementation of a standard for use in different specified environments'.

All quality attributes and their classification are listed in Table 4. The column 'similar meaning' is representing the quality attributes that were combined. Italic items are included in the model.

\section{Conclusions}

We take one step back and recapitulate the research questions:

- What structured set of quality attributes determine the quality of a semantic IS standard? We have used the process of design science to construct a quality model which has been partly validated and improved based on expert opinions gathered through a survey. This structured set, or model, contain quality attributes that can be used to determine the quality of a semantic IS standard. 
- What can we learn from other disciplines, like software engineering or product engineering? A comprehensive list of quality attributes collected from different fields of research has been created and used for setting up the model specific for semantic IS standards.

The importance of this study lays in the fact that no quality model for semantic IS standards was present to date. This research provides a first step towards constructing such a quality model. The results show that quality attributes from different areas of research are not always compatible with semantic IS standards, but still gives valuable input and starting point for setting up a quality model for a new domain, like semantic IS standards.

\section{$7.1 \quad$ Future work}

Future work can be focused on multiple parts. First, our final model and new definitions should be validated. Another focus of future work could be directed to finding suitable measurements for each quality attribute. To use this model in practice you can always ask someone for example how complete the specification is. But how do you measure completeness in a specific situation? Maybe it is complete if it provides the minimalistic functions to operate. But other standards might consider all possible functions thinkable as a complete standard. Also different environments where the standard is implemented can differ quite a lot. A qualitative good standard in one industry might be organised by a closed organisation, while a qualitative good standard in another industry is organised by an open community. The measurement itself is not enough; the scales are of equal importance, with different meanings for different uses. However, we should not forget the goal of increasing interoperability. A generic quality model for semantic IS standards should be seen as a guide to improve interoperability. When application of the model leads to some improvements into the standard that will lead to interoperability improvement, we are one step closer to our goal.

\section{References}

Alexander, J.E. and Tate, M.A. (1999) Web Wisdom: How to Evaluate and Create Information Quality on the Web, 1st ed., L. Erlbaum Associates Inc., Hillsdale, NJ, USA.

Boehm, B.W. (1973) Characteristics of Software Quality, Thompson Ramo Wooldridge Systems Group, Systems Engineering and Integration Division, Redondo Beach, CA.

Brunnermeier, S.B. and Martin, S.A. (2002) 'Interoperability costs in the US automotive supply chain', Supply Chain Management, Vol. 7, No. 2, pp.71-82.

Cavano, J.P. and McCall, J.A. (1978) 'A framework for the measurement of software quality', ACM Sigsoft Software Engineering Notes, Vol. 3, No. 5, pp.133-139.

Crosby, P.B. (1979) Quality is Free: The Art of Making Quality Certain, McGraw Hill, New York.

Dedeke, A. (2000) 'A conceptual framework for developing quality measures for information systems', in Proceedings of 5th International Conference on Information Quality.

Delen, G.P.A.J. and Rijsenbrij, D.B.B. (1992) 'The specification, engineering, and measurement of information systems quality', The Journal of Systems and Software, Vol. 17, No. 3, pp.205-217. 
DeLone, W.H. and McLean, E.R. (2003) 'The DeLone and McLean model of information systems success: a ten-year update', Journal of Management Information Systems, Vol. 19, No. 4, pp.9-30.

Dromey, R.G. (1995) 'Model for software product quality', IEEE Transactions on Software Engineering, Vol. 21, No. 2, pp.146-162.

Dromey, R.G. (1996) 'Cornering the chimera', IEEE Software., Vol. 13, No. 1, pp.33-43.

Folmer, E. et al. (2009) 'Top IS research on quality of transaction standards: a structured literature review to identify a research gap', in Proceedings of the 6th International Conference on Standardization and Innovation in Information Technology SIIT, Verlagshaus Mainz GMbH Aachen.

Folmer, E. et al. (2010a) 'Requirements for a quality measurement instrument for semantic IS standards', in EURAS Proceedings 2010 Service Standardisation, Verlagshaus Mainz GmbH Aachen.

Folmer, E., Luttinghuis, P.O. and Hillergersberg, J.v. (2010b) 'Do semantic IS standards lack quality? A survey among 34 semantic IS standards', [unpublished].

Garvin, D.A. (1984) 'What does 'product quality' really mean?', Sloan Management Review, Vol. 26, No. 1, pp.25-43.

Grady, R.B. (1992) Practical Software Metrics for Project Management and Process Improvement, Prentice-Hall, Inc., Upper Saddle River, NJ, USA.

Hevner, A.R. et al. (2004) 'Design science in information systems research', MIS Quarterly, Vol. 28, No. 1, pp.75-105.

Kahn, B.K., Strong, D.M. and Wang, R.Y. (2002) 'Information quality benchmarks: product and service performance', Commun. ACM, Vol. 45, No. 4, pp.184-192.

Katerattanakul, P. and Siau, K. (1999) 'Measuring information quality of web sites: development of an instrument', in Proceedings of the 20th International Conference on Information Systems, pp.279-285, Association for Information Systems, Charlotte, North Carolina, USA.

McCall, J.A., Richards, P.K. and Walters, G.F. (1977) 'Factors in software quality', Nat'l. Tech. Information Service, Vols. 1, 2 and 3.

Naumann, F. and Rolker, C. (2000) 'Assessment methods for information quality criteria', in Proceedings of the International Conference on Information Quality (IQ).

O'Brien, L., Merson, P. and Bass, L. (2007) 'Quality attributes for service-oriented architectures', in Proceedings of the International Workshop on Systems Development in SOA Environments, IEEE Computer Society, p.3.

Rukanova, B. (2005) 'Business transactions and standards: towards a system of concepts and a method for early problem identification in standa[r]d implementation projects', Enschede, p.262.

Shanks, G. and Corbitt, B. (1999) 'Understanding data quality: social and cultural aspects', in Proceedings of the 10th Australasian Conference on Information Systems.

Spivak, S.M. and Brenner, F.C. (2001) Standardization Essentials: Principles and Practice, CRC Press, New York.

Stvilia, B. et al. (2007) 'A framework for information quality assessment', Journal of the American Society for Information Science and Technology, Vol. 58, No. 12, pp.1720-1733.

Van Zeist, R.H.J. and Hendriks, P.R.H. (1996) 'Specifying software quality with the extended ISO model', Software Quality Journal, Vol. 5, No. 4, pp.273-284.

Wand, Y. and Wang, R.Y. (1996) 'Anchoring data quality dimensions in ontological foundations', Communications of the ACM, Vol. 39, No. 11, pp.86-95.

Wang, R.Y. and Strong, D.M. (1996) 'Beyond accuracy: what data quality means to data consumers', Journal of Management Information Systems, Vol. 12, No. 4, pp.5-34.

Zhu, X. and Gauch, S. (2000) 'Incorporating quality metrics in centralized/distributed information retrieval on the World Wide Web', SIGIR Forum (ACM Special Interest Group on Information Retrieval), pp.288-295. 